\title{
NASA Microgravity Solidification Experiments for the International Space Station
}

NASA Research Associate Report

\author{
Justin McElderry ${ }^{1}$, Micah Hardyman², Tristan Phillips ${ }^{3}$, James Ragan ${ }^{4}$, Rahul Rameshbabu ${ }^{5}$ \\ ${ }^{1}$ Department of Mechanical Engineering, Milwaukee School of Engineering, Milwaukee, WI; \\ ${ }^{2}$ Department of Mechanical Engineering, Virginia Polytechnic Institute and State University, \\ Blacksburg, VA; ${ }^{3}$ Department of Electrical and Computer Engineering, University of Alabama, \\ Tuscaloosa, AL; ${ }^{4}$ Department of Aeronautics \& Astronautics, University of Washington, Seattle, \\ WA; ${ }^{5}$ Department of Aerospace Engineering, Georgia Institute of Technology Atlanta, GA
}

Experiment support offered by the NASA Marshall Space Flight Center Engineering Materials Section, Alabama Space Grant Consortium, and Wisconsin Space Grant Consortium

\begin{abstract}
MMaJIC (Microgravity Materials Joining Investigation Chamber) is a modular experiment environment for performing materials science investigations. MMaJIC provides a controlled and sealed test environment for a wide variety of soldering and brazing experiment to examine porosity in microgravity. MMaJIC's modular cartridge design includes a tray for housing the solder experiments and demonstrates simplicity to astronauts. SoLIDD (Solid Liquid Interface Directional Device) is a device that is used to test samples for directional solidification experiments. Directional solidification is the method of controlling the microstructure of materials. Solidification is useful for creating higher strength materials for space casting. SoLIDD has capability for varying temperature gradient and growth velocity. These quantities utilize heaters, coolers, and a directional drive unit. Two high resolution cameras capture high resolution video of both experiments and thermocouples record live-stream temperature data. Both devices are approved by NASA Headquarters to be contracted to the International Space Station.
\end{abstract}

\section{Introduction}

Gravity-based phenomena drive all ground-based melting and solidification processes. Buoyancy leads to convection and tends to sort layers of a fluid by mass. Fluid shapes are a balance of surface tension, wetting and gravity, and gravity-driven settling is often observed. In microgravity, secondary terms such as thermocapillary-driven convection take a primary role in governing microstructural development. Since these processes are fundamental to routine manufacturing and repair methods such as soldering or brazing, a better understanding of melting and solidification in

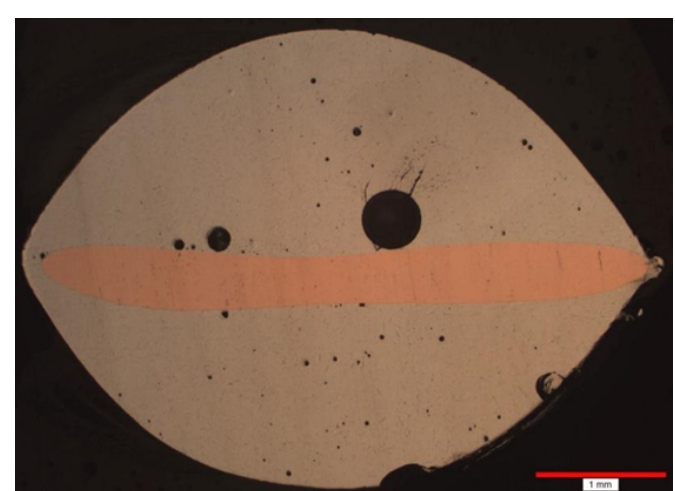

Figure 1: Porosity in microgravity soldering microgravity is essential to any long-duration exploration efforts. Previous studies of in-space soldering have shown that normal ground techniques result in joints that have higher porosity, reduced structural integrity, and suboptimal electrical and thermal conductivity shown at a microscopic view in Figure 1. Further studies of solid/liquid interfaces and phase changes in microgravity are needed to solve problems in applications like these (Daly 2017). 
To meet the need for more studies, the Microgravity Materials Joining Investigative Chamber (MMaJIC) was proposed for flight on the International Space Station (ISS) and was designed as a flexible platform for processing a wide variety of material science payloads. Each sample was prepared in advance on the ground, fitting into a cartridge that is inserted into the on-orbit housing. Parameters for each experiment were encoded into an on-cartridge SD card, which was then read by MMaJIC's microcontroller and used to control the voltage supplied to three power lines, which were primarily used to control the temperature of heating elements along a specified thermal profile. Built-in thermocouples record temperature from each sample and provide the feedback needed for control. These data were logged to the built-in SD card and sent back with the processed sample. The entire operation requires minimal crew input, using a one-button interface to start each sample. MMaJIC provides an environment to perform high-quality science, with minimal operation time and high sample throughput for future research.

The objective of Solid Liquid Interface Directional Device (SoLIDD) was to continue the investigations of directional solidification while testing a wider variety of samples more efficiently using both a furnace and an electric cooler. This allowed SoLIDD to control the thermal gradient of a Sample Ampoule Assembly (SAA) to a wide range of temperatures. With control of the gradient, the SAA translated at a controlled rate, moved the solid/liquid interface along the sample, and drove directional solidification. Real-time imaging and control allowed investigators to examine the dynamics of solidification in microgravity via ISS downlink. The apparatus was designed to utilize the Maintenance Work Area (MWA) for quick accessibility on the ISS compared to the large lead time from the Microgravity Science Glovebox (MSG) (Spivey 2003).

\section{Materials and Methods}

MMaJIC and SoLIDD were initial prototypes units that opened new opportunities for testing solidification with new features. The Guidelines of Risk Management were considered in the decision of specifications for each experiment to list the severity of any issues that arise. (Costello 2014). Specific expectations were organized by subsystems: mechanical, electrical, and software/controls located in Table 1 of Appendix A.

The mechanical design for MMaJIC had been improved to accommodate more diverse experiments and provide a completely adaptive environment for future principal investigators (PI). To achieve this, nearly every component of the original version was redesigned. Primarily, this changed four systems: tray dimensions, housing dimensions, sealing, and lighting. To ensure that future PIs could perform several different experiments with MMaJIC, the tray

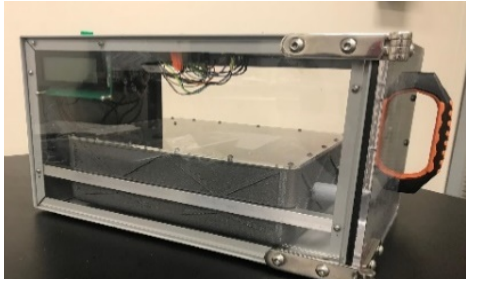

Figure 2: Assembly of MMaJIC. dimensions were increased. Precisely, the overall tray size was changed to 12.4 " in total length, 8.35" in total width, and 3.4" high as shown in Figure 2. Given the improvement in size, trays now accommodated experiment packages up to 9.3 " in length, 7.15 " in width, and 3" in height, for a total of 199.5 cubic inches instead of the trays only containing experiments 4.8 " wide, 0.52 " high, and 5.4" long with a total of 13.5 cubic inches. In practice, roughly about 10.6 square inches or 31.8 cubic inches were devoted to an electronics bay; this was flexible and can be easily moved to any position within the tray. With the much larger tray size, effort had been put in to ensuring that the handle, plug, and rails were centered and inline to prevent any torque on the plug when removing or inserting trays. 
Another notable improvement was the increased plug size that allowed for more voltage lanes and data throughput. Again, this motivated the alignment as it did require slightly more force to insert and remove. Other improvements included modifications to improve overall tray flexibility. Some trays were built with additional capability to allow for convective cooling, piezoelectric coolers, additional sample positions, and even motorized sample manipulation.

The overall size of MMaJIC's housing is now 10.19" wide, 8" high, and 14.88" long to incorporate the size of the new tray; the unit was still able to fit comfortably within the volume of the Maintenance Workbench Area (MWA) as shown in Figure 3. Another benefit was that many of the components were modular for repair. With the new dimensions, camera selection was more diverse and refined with more clearance to put in a higher quality camera. MMaJIC required two levels of containment and these had been refined with several options available. Several designs were

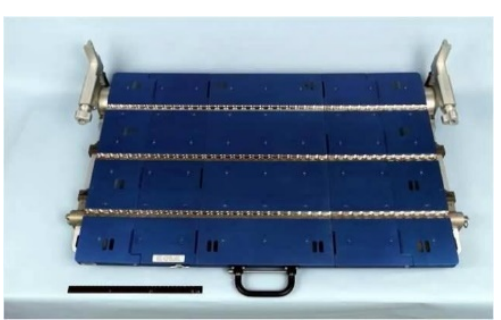

Figure 3: Maintenance Work Area (MWA) on the ISS. used for the tray level of containment. Rubber gaskets were first used to seal the acrylic panel to the top of the tray and epoxy to seal the exits for the wires. The next iteration used open cell neoprene strips for the top seal and silicone caulk for the sealing of wire exits, gaps, and windows. Thick neoprene strips were installed for the door as the second layer of containment.

The electronic modifications to MMaJIC allowed the system to be more adaptable and provided an environment to perform many different types of experiments. The unit was designed to accept anywhere between $12 \mathrm{~V}$ and $30 \mathrm{~V}$ DC power with a maximum allowed current of $10 \mathrm{~A}$. Since each new tray changes, the equivalent resistance of the unit the current required at a given voltage was tray dependent. These power requirements also satisfied the capabilities for the Utility Outlet Power (UOP) as shown in International Space Station Familiarization, with MMaJIC having the same characteristics as a DC power source aboard the ISS in the MWA.

The circuit design was like the previous version of MMaJIC, where the entire unit was controlled with one button with all the safety monitoring, heating control and data collection being completely autonomous by the onboard microcontroller, while a new larger LCD screen provides all vital information quickly to the user. Improvements in circuit protection included placing fuses in an easy to access centralized location on the box's housing with a $10 \mathrm{~A}$ fuse to prevent the unit from drawing too much power from the UOP and a 0.5 A fuse to protect the microprocessor and I/O boards from and unforeseen spikes in current.

The electronics were designed to allow for the housing to function independently of the tray, remaining in standby until it detected that an experimental tray had been inserted. Once inserted, the tray provided the microcontroller with the gains and parameters needed to perform its experiment. While the experiment was running the microcontroller continually monitored temperature and particle sensors for potential errors and auto shutdown conditions. Once the experiment was completed and cooled, the housing returned to standby with the data and processed samples stored on the tray, which could now be safely removed by the user and a new experiment processed. 
The software change to MMaJIC was that an active control system provided flexibility needed to process samples ranging from directional solidification of a variable cross-section to brazing simulations for repairing micro meteors impacts. Using a Proportional Integral Derivative (PID) controller, the housing microcontroller imposed a predefined temperature profile on the samples undergoing testing.

The controller input was an averaged temperature from each of the three heating (or cooling) lanes that were active in the sample. This input temperature was compared to the desired setpoint given by an experimenter set parameter file read by the microcontroller before testing starts. The error between measured and desired temperatures was computed, and the current error, integral of all error and the difference between the current and previous error value were used to compute the controller output. How much each of these terms influence the output was determined by their respective gains, values also encoded by the experimenter before flight on the SD card. The computed output drives each heating lane relay, using the microcontroller's PWM capability to produce pseudo analog results.

During a phase transition (a nonlinear process) control was less precise. During this experiment, linear control of the heating curve was demonstrated for two samples of different thermal mass and heater sizes. For both samples, control within measurement precision was achieved during heating, after accounting for the consistent offset. However, as the samples begin to melt, the desired heating rate could not be realized, as the single sample took $50 \pm 10$ seconds to fully melt, and the temperature remains nearly constant during this transition, resulting in the observed shoulder in the plot. After melting was achieved, the accumulated error results in the controller overshooting the equilibrium temperature slightly before settling. With more thermal mass, the three-sample heating lane took longer to fully melt but does not overshoot the equilibrium temperature. In both cases, the target temperature was set to 193 degrees Celsius to achieve the desired equilibrium of 190 degrees Celsius after accounting for the consistent offset. Also of note was the loss of control during cooling at the phase transition, and the further loss of control as the setpoint attempts to impose a more rapid cooling rate than was achievable. This results from a lack of active cooling in the sample tray. The maximum cooling rate was from the ambient air when the heater is off.

To solve the issue of control loss during a phase transition, the imposed temperature profile was changed to gradually cross the phase transition, allowing the sample time to melt without accumulating error in the controller. A transition rate of 0.2 degrees Celsius/second was found to result in errors within 1\%, after accounting for the offset in temperature. By allowing the setpoints input to the controller as well as the gains of each heating lane to be customized for each experiment processed, MMaJIC provided custom control for each sample. While the response of each sample must be determined experimentally, and the gains tuned manually, this was performed on the ground by the experimenter, and does not require any crew or station time. Once a temperature profile had been decided on, the controller automatically processes the sample without any additional input needed from the crew. This automation allows for arbitrarily complex experiments without an increased burden on station resources. 
To support the new control architecture, the software was rewritten to allow parameters the read off the sample tray SD cards to modify the experiment's operation. Two files included on the SD card specify the gains for controlling each heating lane as well as point out the desired temperature profile for each lane. The software then interpolated between each parameter set point and gave this as input into the controller. This allowed for the custom temperature profiles needed for each experiment to be set in advance by an experimenter on the ground without needing to change the programming of MMaJIC's microcontroller. In addition to flexibility with the experimental parameters, the software incorporated improved safety features, including safety shutoff conditions for each heating lane in the event any thermocouple is $15 \%$ or above the maximum desired temperature in that lane. Combined with a more informative user interface that displayed each heating lane temperature in real time to the operator, MMaJIC gave the operator a quick overview of the system's status and could operate autonomously and safely.

The mechanical design for SoLIDD (as shown in Figure 4) was developed to operate solidification experiments safely and efficiently to improve sample tube testing and offer a broader range of samples for future investigations. The mechanical design was further divided into cooler, furnace, directional drive unit, and electronics subsystems. Combined, these allowed SoLIDD to achieve the directional solidification and temperature gradients required by an experiment.

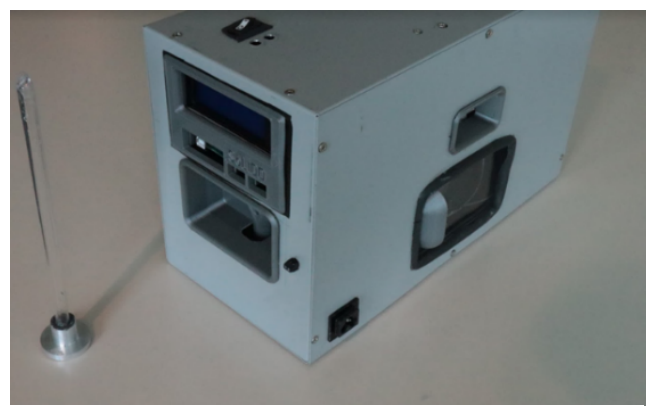

Figure 4: Assembly of SoLIDD.

The cooler unit was designed to create a cold zone for the SAA. An electric cooler (TE-Tech CP$040 \mathrm{HT}$ ) was purchased with a stand to offer a cold range between -17 degrees Celsius to 22 degrees Celsius that utilized a low current. To maximize performance of the cold zone, milled brackets were designed to surround the sample and optimize heat transfer from the sample. The brackets were analyzed in thermal steady state Finite-Element Analysis (FEA) in ANSYS. With the option of multiple sample sizes and geometries, the cooler unit could swap out brackets to fit any geometry the investigator desired. With sliding doors installed on the side panels of the apparatus, a hand could be inserted into the apparatus to exchange brackets with thumbscrews. Insulation was installed between the brackets and thumbscrews for maximum thermal efficiency. Another feature included was carbon fiber felt which was wrapped inside each bracket to maximum contact and increase heat transfer, as carbon fiber has an excellent thermal conductivity. Also installed was the vents for the electric cooler where ambient air was drawn into a fan by the cooler, and the exhaust heat was released from fins on the side of the cooler to provide proper airflow as described and shown in Figure 5.

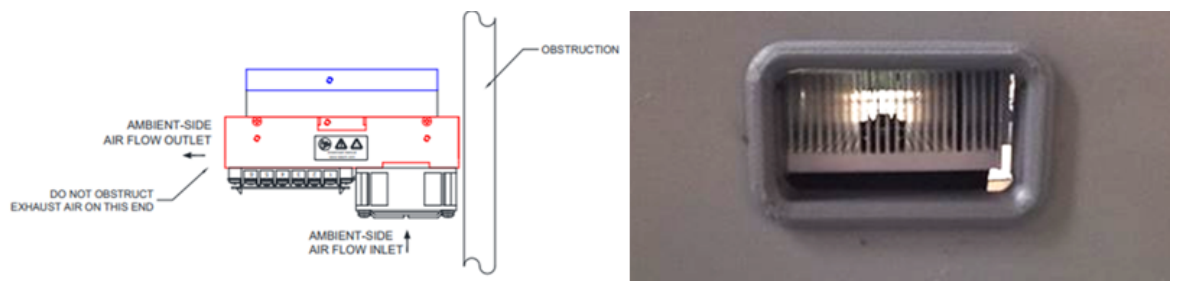

Figure 5: Air flow diagram for cooler and vent developed. 
The furnace unit could be set to a temperature between 22 degrees Celsius and 500 degrees Celsius. Boron nitride was used as the furnace tube and wrapped in Kanthal A-1 wiring. With temperatures being extremely high, insulation was needed to protect the surrounding wire. A half inch of alumina ceramic fiber cotton was wrapped around the furnace tube, and the full assembly was contained in quartz tubing. The insulation lowered the outside temperature to 60 degrees Celsius while the furnace tube was controlled at 500 degrees Celsius. Thermal FEA and testing of the tube demonstrated efficient heat transfer between the furnace and SAA without the need for thermal contact. The furnace was mounted on a stainless-steel mount attached to the top of the apparatus and away from the motor and electronics bay.

The directional drive unit (DDU) consisted of a linear actuator assembly and a stepper motor. The DDU provided the translation needed for directional solidification to occur and positions the sample before and after processing was completed. It was mounted on the inside bottom face of the apparatus. A mount was 3D printed to hold the SAA concentric with the cooler and furnace brackets. To attach the SAA to this mount, the user only needed to insert the sample and tighten the thumbscrew. Once the desired heater and cooler temperatures were achieved, the DDU moved the sample to the location needed throughout for experiment at a rate of 1 micron per second.

The electronics bay stored the electronics of SoLIDD and was mounted on the ceiling of the apparatus. The electronics bay contained a microprocessor, circuit board, controller, LCD screen, on/off switch, and status LEDs. The switch, LEDs, and LCD screen are mounted on the top for clear visibility of the status of the experiment. Holes were built into the side to pass through wiring and give access to a fuse. Power was interfaced through a side panel for simple setup in the MWA. The electrical systems of SoLIDD provided control of the temperatures of both cooler and furnace units, the speed of a directional drive unit, and implement a user interface for the experiment.

The temperature control of the heating and cooling zones were accomplished by calculating a duty cycle using the microcontrollers on-board PID control. Once the output was calculated, the program independently changed the duty cycle of two solid state relays to provide the desired power to the furnace and cooler. Accurate and fast temperature data acquisitions from thermocouples in the furnace and cooler was required for temperature gradient control. Temperature data were acquired using the Adafruit Max 31855 thermocouple breakout board and k-type thermocouples. These data were used as feedback for the controller as it varies the pulse

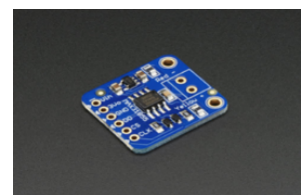

Figure 6: SoLIDD

Breakout board frequency of the relays to drive the system to different temperatures. A model of the breakout board used is shown in Figure 6. Safety components include a fuse, breaker, and voltage regulator. A 2 A fuse connected the $24 \mathrm{~V}$ power supply to the stepper motor driver. The voltage regulator steps down the $24 \mathrm{~V}$ power into $12 \mathrm{~V}$ power usable by the Arduino, a 0.5 A breaker was connected in series with the microcontroller to protect it from unforeseen spikes in current.

The software for SoLIDD consists of an Arduino and a MATLAB program. The Arduino program controlled the furnace, cooler, LCD Screen, and DDU. The MATLAB program controlled a Graphical User Interface (GUI) with status updates, data saving, and high-level control. Figure 7 is a high-level flow chart of the code. The software was divided into four main files in addition to the top level SoLIDD file: Control, Data Logging, IO, and DDU. The Control file contains the functions to implement PID control for the furnace and cooler onboard SoLIDD. 
This code made use of the Arduino PID library to heat and cool to any set point within the maximum and minimum values of each device. The Data Logging file contained the functions to $\log$ data from thermocouples onboard the furnace unit, cooler unit, and SAA. The IO file contained the functions to implement status updates using the LCD screen and onboard LEDs. It displays testing parameters including sample velocity, sample temperature, experiment time, and experiment distance. The DDU file contains the functions to implement the motion of the DDU. The drive unit will move only when the desired temperatures were reached. This file also included limit switch functionality, which ensured that the sample did not exceed the maximum travel distance. When the maximum distance was reached, the sample translates back to the starting position and stopped until a new test began.

The MATLAB GUI worked with three main functions; an opening function, a go button call-back, and a stop button callback. The opening function initialized serial communication with the Arduino Mega. The go button call-back sent furnace temperature, cooler temperature, and growth velocity parameters to the Arduino Mega. The go button call-back also received incoming sample temperature and time-stamp data from the Arduino Mega and graphed it in a realtime plot. The stop button call back ended data transmissions and data receptions, cleared the live plot, and saved sample temperature data and time data for postprocessing.

\section{Key Results}

MMaJIC had already been used to process several ground samples that served as examples for the planned microgravity investigations. Emphasis had been placed on demonstrating the ability to process a wide variety of samples autonomously throughout the desired heating profile while acquiring critical temperature data and demonstrating consistent control. SM2 samples were first used to test the control algorithms during heating and cooling, demonstrate the ability to set parameterized temperature profiles, and linearly increase and decrease the desired temperature rates. In addition, experiments were done to determine the maximum heating rate of the sample. The temperatures for this experiment were shown in Figure 8, where the melting point of the leadtin eutectic could be seen near 183 degrees Celsius during both the heating and cooling portions of the profile. Note that controlled cooling was demonstrated in the liquid lead phase for both samples until control is lost during the phase transition. After the phase transition, an overshoot was observed before each sample resumes cooling along the imposed linear profile. This overshoot suggested that the proportional and integral gains should be lowered for each sample. However, the response remained stable and demonstrated that the desired cooling control could be maintained; in this case easily to the desired lower temperature. 


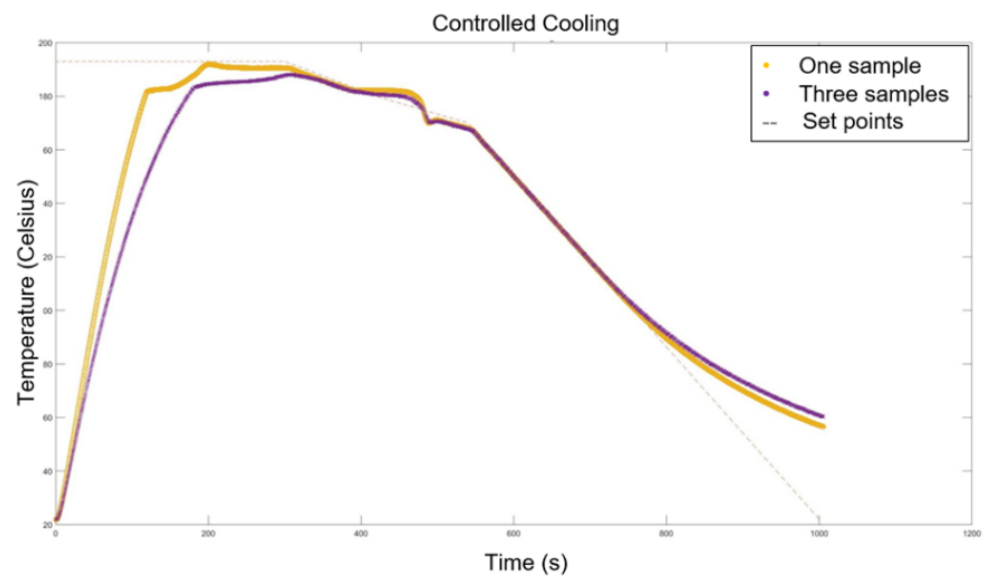

Figure 8: MMaJIC controlled cooling data collection

During initial testing of the ground prototype, SoLIDD successfully developed a solid/liquid interface in a water sample when the cooler was set to -17 degrees Celsius and the furnace was left unpowered. The interface was established in the gradient zone, as could be seen in Figure 9, indicating that the desired interface location was achievable. These results were in good agreement with the FEA modeling of the furnace and cooler set up, also seen in Figure 9, which suggested the interface should be much closer to the cooler than the furnace. While the actual interface was somewhat further from the cooler than the model suggested, this was likely due to a colder set temperature and better insulation than modeled.

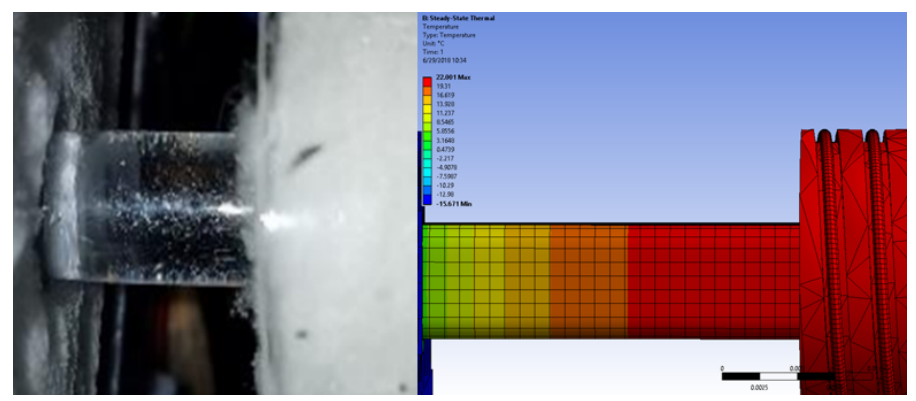

Figure 9: Success in solidification from SoLIDD and Steady-State Thermal FEA comparison.

This early correlation between FEA model and experimental results suggested that with some refinement, FEA modeling could analytically predict the needed furnace and cooler temperatures to establish a solid liquid interface at the desired location. This will minimize the needed experiment time to empirically determine the temperatures needed to reach solidification.

Once the set point temperatures had been determined, SoLIDD could achieve and maintain those temperatures with minimal overshoot or undershoot using the on-board PID controllers. Depending on how hot or cold the sample needs to be, the time necessary to establish temperature gradient for the experiment ranges from 1 to 20 minutes. Control had been successfully demonstrated for several different experiments in the prototype unit, with one test seen in Figure 10. The set temperatures were 130 degrees Celsius for the furnace and 5 degrees Celsius for the cooler. After the set temperatures had been achieved within the tolerance of 1 degree Celsius for five seconds, the DDU translated the sample to its starting position and begins the directional solidification. 


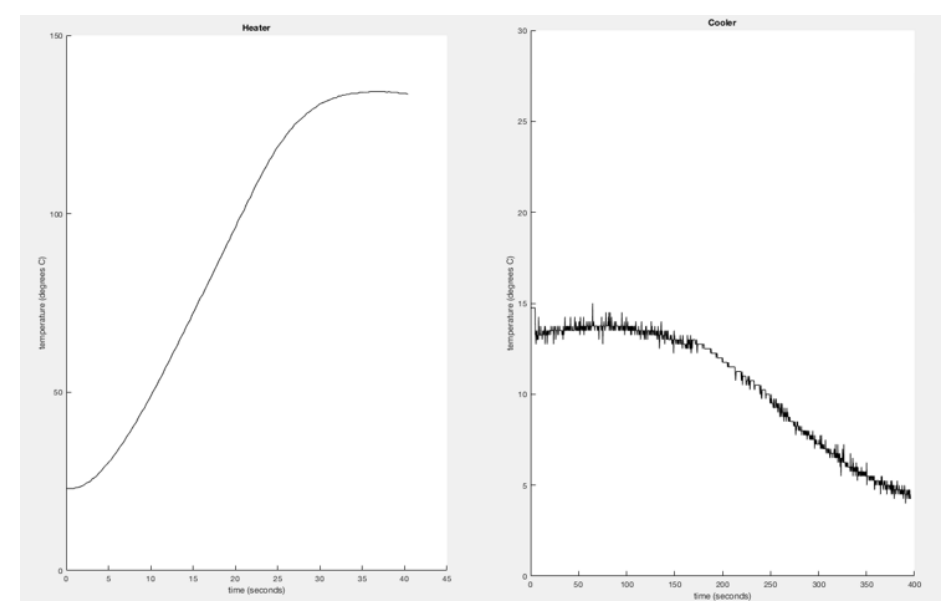

Figure 10: MATLAB plots monitoring live-stream temperature control data from SoLIDD.

\section{Discussion}

MMaJIC was a functional prototype and ground demonstration unit. The successful testing of the SM2 and MSRR/SCA Varying Cross-sections samples indicates that MMaJIC can successfully provide control and data acquisitions for a range of experiments and temperature profiles, providing the needed flexibility to quickly and efficiently process many experiments on orbit. Physically, MMaJIC meets all the design requirements, providing a self-contained housing for processing each experiment. The operation is simple and intuitive, only requiring the ISS operator to supply power, exchange trays and press start. By simplifying and automating its design and operation, MMaJIC has successfully minimized the required station and crew resources. Investigations outside of materials science could include sample trays housing combustion and fluids experiments, particle motion studies requiring onboard video capabilities, or, perhaps, including a linear actuator for directional solidification studies. MMaJIC's adaptability allows for experiment trays to be developed after the initial launch, extending its lifetime and versatility beyond traditional payload.

SoLIDD was also a functional prototype and ground demonstration unit. Ground testing had demonstrated the ability to create a solid liquid interface, collect temperature data, and translate a sample at micron per second rates as needed for a microgravity experiment. A wide range of sample geometries can be accommodated by SoLLID to allow for a variety of experiments and materials to be processed within the same payload. SoLIDD streamlines on-orbit setup and operation, allowing for more rapid sample processing and minimizing crew time and training required. With active control and a GUI that allows the hot and cold zone temperatures to be updated and provides real-time plotting, SoLIDD's user interface allows for downlink to an experimenter on the ground for monitoring and live adjustments. In future space hardware iterations of SoLIDD for contractors, improvements will be made to the structure and ventilation to conform to flight hardware requirements. At the same time, modifications will be made to the housing to mount an internal camera lens system, providing microscopic scale video capture of the solidification process in the investigation. With these upgrades, SoLIDD will be able to test models of solid growth and crystal structures, delivering the same quality of science as previous experiments with more capabilities. 


\section{Acknowledgments}

The SoLIDD and MMaJIC team would like to thank Dr. Richard Grugel (EM-31) for his support as a mentor, Jeff Quick (EM-31) for machining some components in SoLIDD, Ellen Rabenberg (EM-31) for part orders, Taylor Waddell (ES-62) for the assistance and access to the Makerspace in laser cutting and 3D printing, Dr. Louise Strutzenberg (ER-42) for her assistance, and Carey Harris for engineering assistance. Luke Scharber (ER-41) provided electronics parts and Arduinos. The team made use of the Arduino PID library. The team wanted to thank the Alabama Space Grant Consortium and the Wisconsin Space Grant Consortium for the funding the team members work and transportation, Marshall Space Flight Center, and the Space Hardware and Robotics Academy for the internship opportunity to work at NASA.

\section{References}

Costello, K., Ferguson, S., and Kinney, S. (2014) Guidelines for Risk Management. Version F, NASA Independent Verification \& Validation Program.

Daly, Shannen, (2017). MMaJIC, an Experimental Chamber for Investigating Soldering and Brazing in Microgravity. Gravitational and Space Research, vol. 5 no. 2, 31, pp. 28-34.

National Aeronautics and Space Administration, Lyndon B. Johnson Space Center. (1998). International Space Station Familiarization. Houston, Texas: National Aeronautics and Space Administration.

Spivey, R., Gilley, S., Ostrogorsky, A., Grugel, R., and Smith, G. (2003). SUBSA and PFMI Transparent Furnace Systems Currently in use in the International Space Station Microgravity Science Glovebox. AIAA 2003- 1362. 41th AIAA Aerospace Sciences Meeting \& Exhibit.

\section{Appendix A}

\begin{tabular}{|c|c|c|c|}
\hline Experiment & Mechanical & Electrical & Software/Controls \\
\hline MМаJIC & $\begin{array}{l}\text { Provide a housing to safely and efficiently } \\
\text { perform a wide variety of microgravity } \\
\text { experiments } \\
\text { Allow for easy exchanging of trays } \\
\text { Have the flexibility to incorporate } \\
\text { mechanical support } \\
\text { Create two levels of containment for the } \\
\text { experiment, one from the housing and one } \\
\text { from the tray }\end{array}$ & $\begin{array}{l}\text { Follow the power requirements set } \\
\text { forth by the ISS Payload Operations } \\
\text { Plug and Play plan } \\
\text { Design a sizable operational overhead } \\
\text { to accommodate trays with relatively } \\
\text { high-power consumption } \\
\text { Insure the housing functions } \\
\text { independently from the tray, and } \\
\text { allow for tray switch-out with the unit } \\
\text { powered on } \\
\text { Provide a variety of data collection } \\
\text { and safety sensors to ensure safe } \\
\text { operation of the payload }\end{array}$ & $\begin{array}{l}\text { Provide quick and accurate control } \\
\text { to each of the voltage lanes } \\
\text { Ability to follow a given } \\
\text { temperature profile allowing the } \\
\text { investigator to control both } \\
\text { temperature levels, duration, and } \\
\text { heating or cooling rates } \\
\text { Maintain control of a sample after } \\
\text { experiencing a nonlinear response } \\
\text { Read all parameters of the } \\
\text { experiment from each tray } \\
\text { individually and record all measured } \\
\text { data back to the tray }\end{array}$ \\
\hline SoLIDD & $\begin{array}{l}\text { Allow a solidification apparatus to safely } \\
\text { and efficiently operate various } \\
\text { microgravity solid/liquid interface samples } \\
\text { Easy access and visibility to screen, } \\
\text { buttons, and LEDs } \\
\text { Quick and simple SAA insertion into } \\
\text { apparatus with one level of containment } \\
\text { Enough clearance for interchangeable } \\
\text { cooler brackets for testing various samples } \\
\text { Proper pathways of ventilation for cooler }\end{array}$ & $\begin{array}{l}\text { Ability to be powered within the } \\
\text { MWA requirements of } 24 \mathrm{~V} \text { and } 10 \mathrm{~A} \\
\text { Must contain LEDs, LCD screen, and } \\
\text { on/off switch for user interface } \\
\text { Must contain necessary hardware to } \\
\text { allow PWM control of current for } \\
\text { furnace and cooler } \\
\text { Follow the power requirements set } \\
\text { forth by the ISS Payload Operations } \\
\text { Plug and Play plan }\end{array}$ & $\begin{array}{l}\text { Robust control of furnace and cooler } \\
\text { temperature with minimal } \\
\text { oscillations and overshoot } \\
\text { MATLAB GUI to update } \\
\text { parameters, view live data, and save } \\
\text { trial data }\end{array}$ \\
\hline
\end{tabular}

Table 1: Design Specifications of MMaJIC and SoLIDD. 